\title{
What Controls the Strength of Snow-Albedo Feedback?
}

\author{
Xin Qu and Alex Hall \\ Department of Atmospheric and Oceanic Sciences, University of California, Los Angeles, Los Angeles, California
}

(Manuscript received 31 May 2006, in final form 30 October 2006)

\begin{abstract}
The strength of snow-albedo feedback (SAF) in transient climate change simulations of the Fourth Assessment of the Intergovernmental Panel on Climate Change is generally determined by the surfacealbedo decrease associated with a loss of snow cover rather than the reduction in snow albedo due to snow metamorphosis in a warming climate. The large intermodel spread in SAF strength is likewise attributable mostly to the snow cover component. The spread in the strength of this component is in turn mostly attributable to a correspondingly large spread in mean effective snow albedo. Models with large effective snow albedos have a large surface-albedo contrast between snow-covered and snow-free regions and exhibit a correspondingly large surface-albedo decrease when snow cover decreases. Models without explicit treatment of the vegetation canopy in their surface-albedo calculations typically have high effective snow albedos and strong SAF, often stronger than observed. In models with explicit canopy treatment, completely snow-covered surfaces typically have lower albedos and the simulations have weaker SAF, generally weaker than observed. The authors speculate that in these models either snow albedos or canopy albedos when snow is present are too low, or vegetation shields snow-covered surfaces excessively. Detailed observations of surface albedo in a representative sampling of snow-covered surfaces would therefore be extremely useful in constraining these parameterizations and reducing SAF spread in the next generation of models.
\end{abstract}

\section{Introduction}

Snow-albedo feedback (SAF) enhances Northern Hemisphere (NH) extratropical climate sensitivity in climate change simulations (Budyko 1969; Sellers 1969; Schneider and Dickinson 1974; Robock 1983; Cess et al. 1991; Randall et al. 1994; Hall 2004) because of two changes in the snowpack as surface air temperature $\left(T_{s}\right)$ increases (Robock 1985). First snow cover shrinks, and where it does it generally reveals a land surface that is much less reflective of solar radiation. Second, the remaining snow generally has a lower albedo because its optical properties are sensitive to $T_{s}$. For example, wet melting snow, more common in a warmer climate, has a lower surface albedo than dry frozen snow (Robock 1980). Also, in a warmer climate the frequency of storms where precipitation falls as snow rather than rain decreases, particularly during the transition sea-

Corresponding author address: Xin Qu, Department of Atmospheric and Oceanic Sciences, University of California, Los Angeles, P.O. Box 951565, Los Angeles, CA 90095-1565.

E-mail: xinqu@atmos.ucla.edu sons when midlatitude atmospheric temperatures are close to freezing. Since snow albedo decreases with snow age, the resulting increase in average snow age will lead to lower average snow albedos (Wiscombe and Warren 1980). These snowpack metamorphosis effects are typically parameterized either with an explicit dependence of snow albedo on surface temperature (e.g., Robock 1980) or with a dependence of snow albedo on snow age (e.g., Wiscombe and Warren 1980). The two feedback loops associated with shrinking snow cover and the metamorphosis of the remaining snowpack are illustrated schematically in Fig. 1. Their interactions with other climate feedbacks are included in a climate feedback diagram given in Robock (1985).

The strength of SAF, including both feedback loops described above, can be quantified by the amount of additional net shortwave radiation at the top of the atmosphere (TOA) as surface albedo decreases in association with a $1^{\circ} \mathrm{C}$ increase in $T_{s}$ [following Cess and Potter (1988)]:

$$
\frac{\partial Q_{\text {net }}}{\partial T_{s}}=\frac{\partial Q_{\text {net }}}{\partial \alpha_{s}} \frac{\Delta \alpha_{s}}{\Delta T_{s}}=-Q \frac{\partial \alpha_{p}}{\partial \alpha_{s}} \frac{\Delta \alpha_{s}}{\Delta T_{s}} .
$$

DOI: 10.1175/JCLI4186.1

(C) 2007 American Meteorological Society 


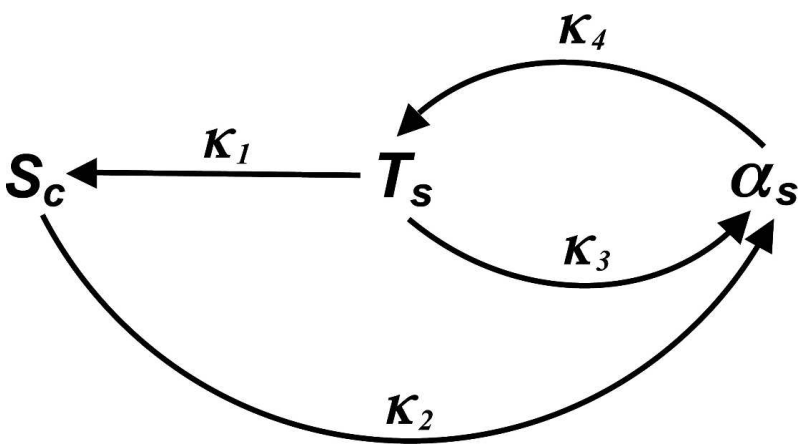

FIG. 1. Schematic drawing of the two feedback loops contributing to snow-albedo feedback. One is the snow cover feedback: an increase in surface air temperature $\left(T_{s}\right)$ leads to a reduction in snow cover $\left(S_{c}\right)$, which in turn leads to a reduction in surface albedo $\left(\alpha_{s}\right)$. The second is the snowpack metamorphosis feedback: the increase in $T_{s}$ leads to a reduction in albedo of the snowpack itself. The reduction in surface albedo resulting from both feedbacks leads in turn to a further increase in $T_{s}$. The meaning of the $\kappa$ symbols is explained at the beginning of section 3 . Units of $\kappa_{1}$ and $\kappa_{3}$ are $\% \mathrm{~K}^{-1}, \kappa_{4}$ is $\mathrm{K}^{-1}$, and $\kappa_{2}$ is dimensionless.

Here $Q$ and $Q_{\text {net }}$ are the incoming and net shortwave radiation at the TOA (where $Q$ is taken to be a constant), $\alpha_{s}$ is the surface albedo, and $\alpha_{p}$ is the planetary albedo. Equation (1) indicates that the strength of SAF is determined by the product of two terms, a coefficient representing the variation in planetary albedo with surface albedo $\left(\partial \alpha_{p} / \partial \alpha_{s}\right)$ and another representing the change in surface albedo associated with a $1^{\circ} \mathrm{C}$ change in the surface air temperature $\left(\Delta \alpha_{s} / \Delta T_{s}\right)$. The magnitude of the second term is determined by the strength of the snow cover and snowpack metamorphosis feedback loops of Fig. 1. SAF is strongest in springtime because both $Q$ and snow cover are large (Robock 1983). In fact, Hall (2004) reports that approximately half the total NH SAF to simulated global climate change occurs during NH spring. Thus we focus on this season in our assessment of the two feedback loops controlling SAF strength.

Recently a highly accurate technique was developed to calculate the coefficient $\partial \alpha_{p} / \partial \alpha_{s}$ given standard model output (Qu and Hall 2006). It was used to calculate springtime $\partial \alpha_{p} / \partial \alpha_{s}$ values in NH land areas for transient climate change experiments of the Fourth Assessment (AR4) of the Intergovernmental Panel on Climate Change (IPCC). The intermodel variation in $\partial \alpha_{p} / \partial \alpha_{s}$ is small, with most models agreeing to within $10 \%$ that a given $\alpha_{s}$ anomaly results in an $\alpha_{p}$ anomaly one-half as large (Fig. 2a).

This agreement occurs because the main factor controlling $\partial \alpha_{p} / \partial \alpha_{s}$ is the transmissivity of the clear-sky atmosphere, where the models' radiative transfer schemes

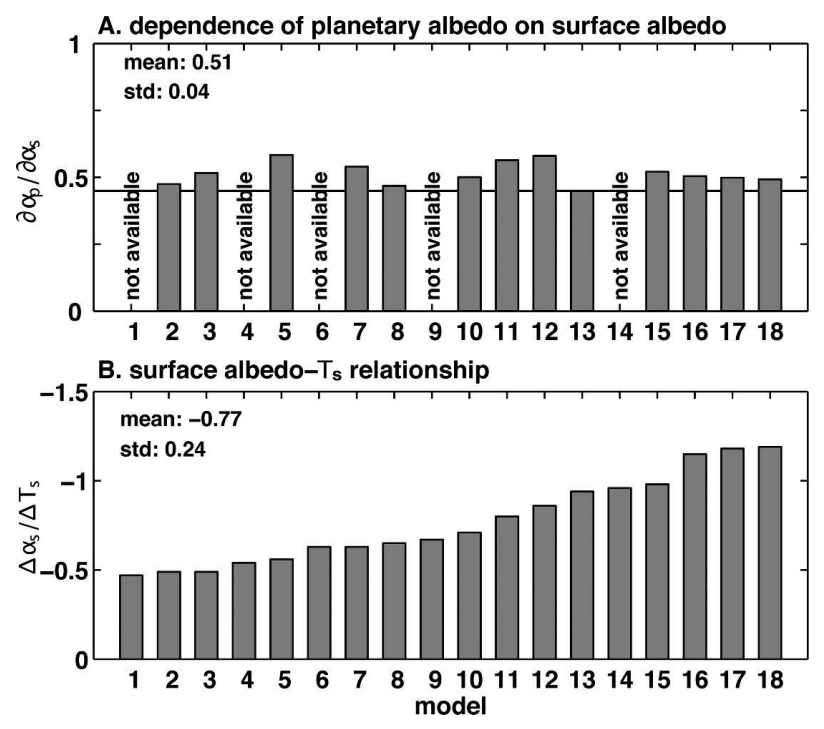

FIG. 2. The climatological springtime mean values of (a) $\partial \alpha_{p} / \partial \alpha_{s}$ in the $\mathrm{NH}$ extratropical land areas in the simulated present-day (1901-2000) climate in 13 AR4 climate models (Table 1), calculated according to the methods described in Qu and Hall (2006). The corresponding International Satellite Cloud Climatology Project (ISCCP) value was obtained through identical procedures for the period of the ISCCP data (1984-2000) and is shown as a solid line. The mean and standard deviation of $\partial \alpha_{p} / \partial \alpha_{s}$ in the 13 models are shown in the top left corner. Note that we did not calculate $\partial \alpha_{p} / \partial \alpha_{s}$ in 5 of the 18 models listed in Table 1 because they did not provide all the necessary variables. (b) The surface albedo and $T_{s}$ relationship $\left(\Delta \alpha_{s} / \Delta T_{s}\right)$ in the $\mathrm{NH}$ extratropical land areas during spring in 18 climate change simulations. The $\Delta \alpha_{s}$ and $\Delta T_{s}$ represent differences in springtime mean surface albedo and $T_{s}$ between the present-day (1901-2000) and future (2100-99) climate. The mean and standard deviation of $\Delta \alpha_{s} / \Delta T_{s}$ in the 18 models are shown in the top left corner. Units are $\% \mathrm{~K}^{-1}$.

converge in their handling of the atmosphere's interaction with solar photons.

While there is consistency from model to model in $\partial \alpha_{p} / \partial \alpha_{s}$ values, the second term in Eq. (1), $\Delta \alpha_{s} / \Delta T_{s}$ diverges widely. We calculate this term in the climate change context based on springtime values of $\alpha_{s}$ and $T_{s}$ averaged over $\mathrm{NH}$ land areas from the beginning and end of the AR4 experiments (Fig. 2b). Not only is there almost a threefold spread in $\Delta \alpha_{s} / \Delta T_{s}$ but there is no clear preference for a central value. An understanding of the controls on SAF strength therefore amounts to a determination of what causes $\Delta \alpha_{s} / \Delta T_{s}$ to vary so much from model to model. This in turn requires an assessment of the relative contributions of snow cover reduction and snowpack metamorphosis to the surfacealbedo reduction. In this study, we develop methods to separate and quantify contributions to $\Delta \alpha_{s} / \Delta T_{s}$ from these two feedback loops.

The causes of the spread of $\Delta \alpha_{s} / \Delta T_{s}$ are particularly important in light of recent work (Hall and Qu 2006), 
TABLE 1. Eighteen models used for AR4 climate change experiments and the description of surface-albedo parameterizations incorporated in the models. The "snow albedo" column describes how snow albedo is calculated, including the way metamorphosis effects are parameterized, either with an explicit temperature dependence or with a dependence on snow age. They are represented in the table by "temperature" and "snow age," respectively. The "vegetation masking effect" column describes how snow albedo is used to calculate surface albedo. In type 1 , surface albedo is calculated based on a radiation transfer scheme between canopy top and ground. Ground albedo is a weighted mean of snow albedo and soil albedo (weights determined by snow cover). In type 2, surface albedo is a weighted mean of vegetation albedo and ground albedo (weights determined by vegetation cover). In type 3 , surface albedo is a weighted mean of snow-free land albedo and snow albedo (weights determined by snow cover). Snow albedo depends on vegetation type. In type 4, surface albedo is a weighted mean of snow-free land albedo and snow albedo (weights determined by snow cover). Snow albedo is independent of vegetation type. Note that models were ordered according to their values of $\Delta \alpha_{s} / \Delta T_{s}$.

\begin{tabular}{|c|c|c|c|c|}
\hline No. & Model & Snow albedo & $\begin{array}{c}\text { Vegetation } \\
\text { masking effect }\end{array}$ & Reference \\
\hline 1 & MRI CGCM2.3.2 & temperature & type 1 & Yukimoto et al. (2006) \\
\hline 2 & CNRM-CM3 & snow age & type 2 & Douville et al. (1995) \\
\hline 3 & CSIRO Mk3.0 & snow age & type 2 & Gordon et al. (2002) \\
\hline 4 & GISS-ER & snow age & type 2 & Hansen et al. (1983) \\
\hline 5 & FGOALS-g1.0 & snow age & type 1 & Yu et al. (2004) \\
\hline 6 & PCM & snow age & type 1 & Oleson et al. (2004) \\
\hline 7 & CGCM3.1 (T47) & snow age & type 2 & Verseghy et al. (1993) \\
\hline 8 & ECHAM5/MPI-OM & temperature & type 2 & Roeckner et al. (2003) \\
\hline 9 & CGCM3.1 (T63) & snow age & type 2 & Verseghy et al. (1993) \\
\hline 10 & UKMO HadCM3 & temperature & type 3 & Cox et al. (1999) \\
\hline 11 & UKMO HadGEM1 & temperature & type 3 & Essery et al. (2001) \\
\hline 12 & MIROC3.2(medres) & snow age & type 1 & Takata et al. (2003) \\
\hline 13 & CCSM3 & snow age & type 1 & Oleson et al. (2004) \\
\hline 14 & ECHO-G & temperature & type 2 & Roeckner et al. (1996) \\
\hline 15 & IPSL CM4 & snow age & type 3 & Chalita and Le Treut (1994) \\
\hline 16 & GFDL CM2.0 & temperature & type 4 & Milly and Shmakin (2002) \\
\hline 17 & INM-CM3.0 & temperature & type 4 & Alekseev et al. (1998) \\
\hline 18 & GFDL CM2.1 & temperature & type 4 & Milly and Shmakin (2002) \\
\hline
\end{tabular}

demonstrating that many models have an unrealistic SAF strength in the context of the present-day seasonal cycle of $T_{s}$. Because of a very strong correlation between the SAF strength in seasonal cycle and climate change contexts, addressing this bias in the seasonal cycle would significantly reduce the spread of SAF strength in climate change. In this study we develop a very simple picture of the reason for the spread in $\Delta \alpha_{s} / \Delta T_{s}$ in the current generation of climate simulations and relate SAF strength to specific choices about surface-albedo parameterizations and tunable parameters within them. This provides the climate modeling community with guidance in creating a more realistic SAF in individual models and ultimately reducing the spread in SAF.

Our study is presented as follows. The AR4 climate simulations and the models' surface-albedo parameterizations are briefly described in section 2, followed in section 3 by a presentation of an idealized mathematical framework relating the two feedback loops controlling $\Delta \alpha_{s} / \Delta T_{s}$ to quantities typically provided in model output. This framework is used in section 4 to separate and quantify the components of $\Delta \alpha_{s} / \Delta T_{s}$ in each model and to determine which components are responsible for its intermodel spread. In section 5, we discuss how SAF strength is related to surface-albedo schemes and tun- able parameters within them. A summary and implications are given in section 6 , followed by an appendix giving a description of the technique used to convert snow mass values outputted by many models to snow cover values necessary for our calculations.

\section{Climate simulations and snow-albedo parameterizations}

The simulated climates of the twentieth and twentysecond centuries were extracted from the "720-ppm stabilization experiment" with 18 AR4 models (see Table 1). In these experiments, historical twentieth-century forcing was imposed, followed by the IPCC's AlB emission scenario for the twenty-first century. At year 2100, anthropogenic forcings were fixed for the remainder of the experiments, which end at year 2200. Though this forcing scenario was imposed on 23 models for the AR4, only the 18 listed in Table 1 had a complete time series of the necessary variables when our analyses were performed.

The schemes taking into account effects of snow on surface albedo can be grouped into four categories according to the way the masking effect of vegetation is parameterized. We list them here in order of decreasing 
complexity. The schemes of type 1 (including 5 models) employ a full-blown canopy radiative transfer model. In type 2 schemes (including 7 models), canopy albedo is prescribed according to vegetation type and then modified depending on whether snow is sticking to the canopy, and the overall surface albedo is a weighted mean of canopy albedo and ground albedo, with weights given by the fraction of vegetation coverage. In both types 1 and 2, ground albedo is given as a weighted mean of snow albedo and soil albedo, with weights determined by snow cover. And in both types, canopy reflectivity is increased when snow is allowed to stick to the canopy. In the simpler schemes of types 3 and 4 (each including 3 models), the canopy and ground albedos are not treated separately, and the overall surface albedo is simply a weighted mean of snow-free surface albedos and snow albedo, with weights determined by snow cover. In the type 3 schemes, the snow albedo depends on vegetation type, while the type 4 schemes are even simpler, with snow albedos independent of vegetation type.

An important difference between the first two and last two types is apparent where the ground is completely covered by snow. In this case, the surface albedo in types 3 and 4 is equivalent to the prescribed snowalbedo values while the surface albedo in types 1 and 2 is probably smaller than the prescribed snow albedo, since the canopy influences the surface albedo and is likely darker than snow. We emphasize this distinction throughout this paper by reserving the term "snow albedo" for the albedo of the snow itself, and referring to the albedo of surfaces with $100 \%$ snow cover as the "effective snow albedo." They are equivalent and both tunable in type 3 and 4 models, while in type 1 and 2 models the effective snow albedo is determined by snow albedo and canopy effects. Table 1 shows the category each model belongs to. Links between the characteristics of snow-albedo parameterizations discussed here and the strength of SAF are examined in section 5 .

\section{An idealized model to understand $\Delta \alpha_{s} / \Delta T_{s}$}

In this section, we introduce a framework to separate and understand the two feedback loops determining the magnitude of $\Delta \alpha_{s} / \Delta T_{s}$ in climate change simulations. In Fig. 1, snow cover, surface albedo, and $T_{s}$ are connected by arrows representing effects of one variable on another. There are four main effects, with $\kappa_{1}, \kappa_{2}, \kappa_{3}$, and $\kappa_{4}$ representing the constant of proportionality translating the magnitude of an anomaly in one variable into the magnitude of an anomaly in the affected variable. The reduction in snow cover induced by a unit increase in $T_{s}$ is given by $\kappa_{1}$, and the change in surface albedo induced by a unit change in snow cover is then given by $\kappa_{2}$. Meanwhile, the $T_{s}$ increase also generates a surfacealbedo reduction of the snowpack itself because of snowpack metamorphosis. The associated constant of proportionality is given by $\kappa_{3}$. Finally, $\kappa_{4}$ gives the change in $T_{s}$ arising from a unit change in surface albedo through heat exchange processes between the surface and atmosphere.

Suppose we have an initial warming, $\Delta T_{s 0}$, arising from an external forcing that sets in motion the interactions of Fig. 1: a decrease in snow cover and hence surface albedo occurs, as well as a decrease in albedo of the existing snowpack, leading finally to an additional increase in $T_{s}$. This incremental $T_{s}$ increase and subsequent increments, represented by $\Delta T_{s 1}, \Delta T_{s 2}, \ldots, \Delta T_{s n}$, can be expressed in terms of $\kappa_{1}, \kappa_{2}, \kappa_{3}$, and $\kappa_{4}$ :

$$
\begin{aligned}
\Delta T_{s 1} & =\kappa_{3} \kappa_{4} \Delta T_{s 0}+\kappa_{1} \kappa_{2} \kappa_{4} \Delta T_{s 0} \\
& =\left(\kappa_{3} \kappa_{4}+\kappa_{1} \kappa_{2} \kappa_{4}\right) \Delta T_{s 0}, \\
\Delta T_{s 2} & =\kappa_{3} \kappa_{4} \Delta T_{s 1}+\kappa_{1} \kappa_{2} \kappa_{4} \Delta T_{s 1} \\
& =\left(\kappa_{3} \kappa_{4}+\kappa_{1} \kappa_{2} \kappa_{4}\right)^{2} \Delta T_{s 0}, \\
\Delta T_{s n} & =\kappa_{3} \kappa_{4} \Delta T_{s n-1}+\kappa_{1} \kappa_{2} \kappa_{4} \Delta T_{s n-1} \\
& =\left(\kappa_{3} \kappa_{4}+\kappa_{1} \kappa_{2} \kappa_{4}\right)^{n} \Delta T_{s 0} .
\end{aligned}
$$

Here, we assumed that $\kappa_{1}, \kappa_{2}, \kappa_{3}$, and $\kappa_{4}$ are constant with each increment. Then, the total change in surface air temperature, $\Delta T_{s}$, can be obtained by summing up the initial perturbation and all the incremental changes:

$$
\begin{aligned}
\Delta T_{s}= & \Delta T_{s 0}+\Delta T_{s 1}+\Delta T_{s 2}+\cdots+\Delta T_{s n} \\
= & {\left[1+\left(\kappa_{3} \kappa_{4}+\kappa_{1} \kappa_{2} \kappa_{4}\right)+\left(\kappa_{3} \kappa_{4}+\kappa_{1} \kappa_{2} \kappa_{4}\right)^{2}+\cdots\right.} \\
& \left.+\left(\kappa_{3} \kappa_{4}+\kappa_{1} \kappa_{2} \kappa_{4}\right)^{n}\right] \Delta T_{s 0} .
\end{aligned}
$$

We assume the series in Eq. (5) converges when $n$ goes to infinity and thus we obtain

$$
\Delta T_{s}=\frac{\Delta T_{s 0}}{1-\left(\kappa_{3} \kappa_{4}+\kappa_{1} \kappa_{2} \kappa_{4}\right)} .
$$

The surface-albedo perturbations associated with each $T_{s}$ perturbation, $\Delta \alpha_{s 0}, \Delta \alpha_{s 1}, \ldots, \Delta \alpha_{s n}$, can be similarly derived:

$$
\begin{aligned}
& \Delta \alpha_{s 0}=\kappa_{3} \Delta T_{s 0}+\kappa_{1} \kappa_{2} \Delta T_{s 0}=\left(\kappa_{3}+\kappa_{1} \kappa_{2}\right) \Delta T_{s 0} \\
& \Delta \alpha_{s n}=\kappa_{3} \Delta T_{s n}+\kappa \kappa_{2} \Delta T_{s n}=\left(\kappa_{3}+\kappa_{1} \kappa_{2}\right) \Delta T_{s n} .
\end{aligned}
$$

The total change in surface albedo, $\Delta \alpha_{s}$, is the sum of all the perturbations

$$
\begin{aligned}
\Delta \alpha_{s} & =\Delta \alpha_{s 0}+\Delta \alpha_{s 1}+\Delta \alpha_{s 2}+\cdots+\Delta \alpha_{s n} \\
& =\left(\kappa_{3}+\kappa_{1} \kappa_{2}\right)\left(\Delta T_{s 0}+\Delta T_{s 1}+\Delta T_{s 2}+\cdots+\Delta T_{s n}\right) .
\end{aligned}
$$


Using Eq. (6), we have

$$
\Delta \alpha_{s}=\frac{\left(\kappa_{3}+\kappa_{1} \kappa_{2}\right) \Delta T_{s 0}}{1-\left(\kappa_{3} \kappa_{4}+\kappa_{1} \kappa_{2} \kappa_{4}\right)} .
$$

Likewise, we can obtain the total change in snow cover $\left(S_{c}\right)$ :

$$
\Delta S_{c}=\frac{\kappa_{1} \Delta T_{s 0}}{1-\left(\kappa_{3} \kappa_{4}+\kappa_{1} \kappa_{2} \kappa_{4}\right)} .
$$

Based on Eqs. (6), (10), and (11), the ratios $\Delta \alpha_{s} / \Delta T_{s}$ and $\Delta S_{c} / \Delta T_{s}$ are written as

$$
\frac{\Delta \alpha_{s}}{\Delta T_{s}}=\kappa_{3}+\kappa_{1} \kappa_{2}
$$

and

$$
\frac{\Delta S_{c}}{\Delta T_{s}}=\kappa_{1} .
$$

Plugging Eq. (13) into (12), we obtain an expression for the SAF parameter in terms of the constants $\kappa_{2}$ and $\kappa_{3}$ :

$$
\frac{\Delta \alpha_{s}}{\Delta T_{s}}=\kappa_{3}+\kappa_{2} \frac{\Delta S_{c}}{\Delta T_{s}} .
$$

Equation (14) is our framework for understanding contributions to $\Delta \alpha_{s} / \Delta T_{s}$. It implies that $\Delta \alpha_{s} / \Delta T_{s}$ is con- trolled by two components, one arising from the metamorphosis of the snowpack in a warmer climate $\left(\kappa_{3}\right)$ and another stemming from the reduction of snow cover $\left(\kappa_{2} \Delta S_{c} / \Delta T_{s}\right)$.

To gain insight into the physical processes represented by $\kappa_{2}$ and $\kappa_{3}$, we introduce the following approximation for surface albedo in snow-covered land areas for present and future climates:

$$
\alpha_{s}^{p}=S_{c}^{p}\left(\alpha_{\text {snow }}^{p}-\alpha_{\text {land }}\right)+\alpha_{\text {land }}
$$

and

$$
\alpha_{s}^{f}=S_{c}^{f}\left(\alpha_{\text {snow }}^{f}-\alpha_{\text {land }}\right)+\alpha_{\text {land }},
$$

where the superscripts " $p$ " and " $f$ " represent the present and future climate. Here $\alpha_{\text {snow }}$ represents effective snow albedo (the albedo of a completely snowcovered surface after taking into account possible canopy effects) and $\alpha_{\text {land }}$ is snow-free land albedo. Note that $\alpha_{\text {land }}$ is a constant, so that we are not considering changes in snow-free land albedo resulting from climate change here. Subtracting Eq. (15) from (16) and then manipulating, we obtain an expression for $\Delta \alpha_{s}$ that is the sum of two terms, one relating to the change in effective snow albedo and the other relating to a change in snow cover:

$$
\begin{aligned}
\Delta \alpha_{s} & =\alpha_{s}^{f}-\alpha_{s}^{p}=\frac{S_{c}^{f}+S_{c}^{p}}{2}\left(\alpha_{\text {snow }}^{f}-\alpha_{\text {snow }}^{p}\right)+\left(S_{c}^{f}-S_{c}^{p}\right)\left(\frac{\alpha_{\text {snow }}^{f}+\alpha_{\text {snow }}^{p}}{2}-\alpha_{\text {land }}\right) \\
& =\frac{S_{c}^{f}+S_{c}^{p}}{2}\left(\alpha_{\text {snow }}^{f}-\alpha_{\text {snow }}^{p}\right)+\Delta S_{c}\left(\frac{\alpha_{\text {snow }}^{f}+\alpha_{\text {snow }}^{p}}{2}-\alpha_{\text {land }}\right) .
\end{aligned}
$$

Dividing Eq. (17) by the change in $T_{s}$ between the two climates, we have

$$
\begin{aligned}
\frac{\Delta \alpha_{s}}{\Delta T_{s}}= & \frac{S_{c}^{f}+S_{c}^{p}}{2} \frac{\alpha_{\text {snow }}^{f}-\alpha_{\text {snow }}^{p}}{\Delta T_{s}} \\
& +\left(\frac{\alpha_{\text {snow }}^{f}+\alpha_{\text {snow }}^{p}}{2}-\alpha_{\text {land }}\right) \frac{\Delta S_{c}}{\Delta T_{s}} .
\end{aligned}
$$

Comparing Eqs. (14) and (18), we obtain the following expressions for $\kappa_{2}$ and $\kappa_{3}$ :

$$
\kappa_{2}=\frac{\alpha_{\text {snow }}^{f}+\alpha_{\text {snow }}^{p}}{2}-\alpha_{\text {land }}
$$

and

$$
\kappa_{3}=\frac{S_{c}^{f}+S_{c}^{p}}{2} \frac{\alpha_{\text {snow }}^{f}-\alpha_{\text {snow }}^{p}}{\Delta T_{s}} .
$$

Equations (19) and (20) indicate that $\kappa_{2}$ may be interpreted as the mean contrast between effective snow albedo (averaged over the present and future climates) and snow-free land albedo. Meanwhile, $\kappa_{3}$ represents changes in effective snow albedo with respect to changes in $T_{s}$, modulated by mean snow cover.

\section{Separating snow metamorphosis and snow cover components}

To quantify and compare the contributions of snow metamorphosis and snow cover components to the reduction in surface albedo $\left(\Delta \alpha_{s}\right)$, represented by the two terms on the right-hand side of Eq. (17), we need to estimate $S_{c}^{f}, S_{c}^{p}, \alpha_{\text {snow }}^{f}, \alpha_{\text {snow }}^{p}$, and $\alpha_{\text {land }}$ for each model. The $S_{c}^{f}$ and $S_{c}^{p}$ can be readily derived from snow mass values outputted by many of the models (see the appendix for details on how this is done), and thus the 
only unknowns are $\alpha_{\text {snow }}^{f}, \alpha_{\text {snow }}^{p}$, and $\alpha_{\text {land }}$. As demonstrated in section 3 , these three variables are constrained by Eqs. (15) and (16). To solve for them, we need a third constraining expression to complement the two equations. In this section, we construct such an expression by estimating values of $\left(\alpha_{\text {snow }}^{f}-\alpha_{\text {snow }}^{p}\right) / \Delta T_{s}$. This term represents the variation of effective snow albedo with temperature, and as demonstrated in Eq. (18), this can be weighted by mean snow cover to give the contribution of snow metamorphosis to $\Delta \alpha_{s} / \Delta T_{s}$.

For simplicity, we denote $\left(\alpha_{\text {snow }}^{f}-\alpha_{\text {snow }}^{p}\right) / \Delta T_{s}$ by $F\left(T_{s}\right)$. Here, the dependence of $F$ on $T_{s}$ accounts for the fact that the snow metamorphosis effect varies significantly with the mean temperature of a snow-covered area undergoing climate change. In general, both the snow melting and aging effects increase as the mean temperature approaches freezing. To quantify $F\left(T_{s}\right)$, we focus on the snow interior, defined as the regions where the surface is $100 \%$ snow-covered in both the current and future climate. In these regions any change in $\alpha_{s}$ stems entirely from the change in the effective albedo of the snowpack itself. Here $S_{c}^{p}=S_{c}^{f}=1$ by definition and thus according to Eqs. (14) and (20), $\Delta \alpha_{s}=F\left(T_{s}\right) \Delta T_{s}$. Since $\Delta \alpha_{s}$ and $\Delta T_{s}$ at every $100 \%$ snow-covered grid point are known from the model output, we can group them by intervals of $T_{s}$ and then average them for each $T_{s}$ interval. Denoting the resultant functions by $\overline{\Delta \alpha_{s}}\left(T_{s}\right)$ and $\overline{\Delta T_{s}}\left(T_{s}\right)$, we can evaluate $F\left(T_{s}\right)$ by dividing $\overline{\Delta \alpha_{s}}\left(T_{s}\right)$ by $\overline{\Delta T_{s}}\left(T_{s}\right)$. We perform the calculation based on climatological monthly mean $\alpha_{s}$ and $T_{s}$ data. This provides samples large enough to achieve stable statistics for most of the 18 simulations listed in Table 1. [The Parallel Climate Model (PCM), ECHAM5/Max Planck Institute Ocean Model (MPI-OM), ECHAM and the global Hamburg Ocean Primitive Equation (ECHO-G), and the Institute of Numerical Mathematics Coupled Model version 3.0 (INM-CM3.0) were not used in this calculation because the PCM did not have either snow cover or snow mass available when our analyses were performed, and ECHAM5/MPI-OM, ECHO-G, and INM-CM3.0 do not have enough $100 \%$ snow-covered grid points to produce stable statistics, particularly at temperatures close to freezing.]

To reveal general characteristics of $F$, we show its ensemble-mean values at different $T_{s}$ in Fig. 3. For $T_{s}$, $F$ is consistently negative, indicating that effective snow albedo decreases as $T_{s}$ increases. The $F$ increases from 250 to $270 \mathrm{~K}$, where it reaches its maximum, signifying the largest snow metamorphosis effect there, and then decays rapidly above this point. The details of each model's $F$ (not shown) vary somewhat, mostly because of differing representations of the snow metamorphosis

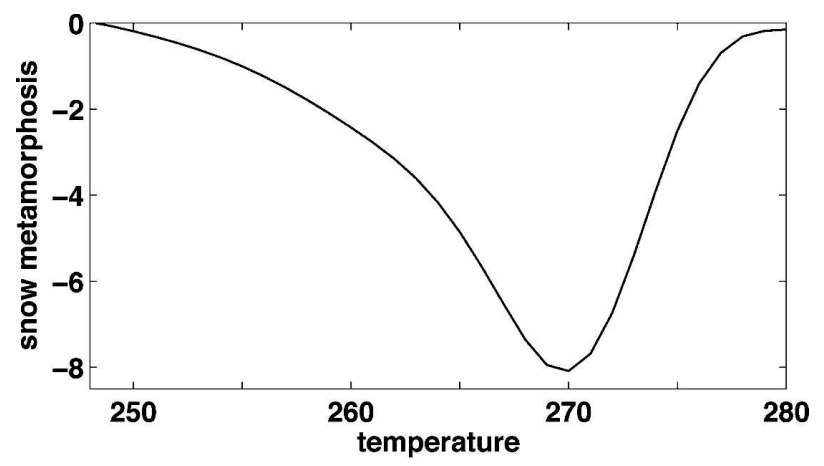

FIG. 3. Ensemble-mean values of $F\left(10^{-3} \mathrm{~K}^{-1}\right)$ as a function of $T_{s}(\mathrm{~K})$ calculated based on $F$ values in the 14 simulations (see text).

effect in the simulations. However, in general they closely resemble the curve shown in Fig. 3.

Once $F$ is known, we have a third expression constraining $\alpha_{\text {snow }}^{f}$ and $\alpha_{\text {snow }}^{p}$ in addition to Eqs. (15) and (16). They form a complete set of equations that we can solve for $\alpha_{\text {snow }}^{f}, \alpha_{\text {snow }}^{p}$, and $\alpha_{\text {land }}$. (The ensemble-mean $F$ values were used in the calculations for ECHAM5/ MPI-OM, ECHO-G, and INM-CM3.0.) Then we can evaluate the change in $\alpha_{s}$ attributable to snow metamorphosis and that attributable to snow cover at each snow-covered grid point using Eq. (17). We then average the 2 contributions to the $\alpha_{s}$ reduction over the $\mathrm{NH}$ land areas poleward of $30^{\circ} \mathrm{N}$ and divide both of them by the $T_{s}$ increase averaged over the same area, giving the 2 components of $\Delta \alpha_{s} / \Delta T_{s}$ for each simulation. The results are shown in Fig. 4. The metamorphosis component is generally small compared to the snow cover component. This reveals that snow-albedo feedback results mostly from the reduction in snow cover rather than the reduction in albedo of the snowpack itself. Not only is the metamorphosis component generally small

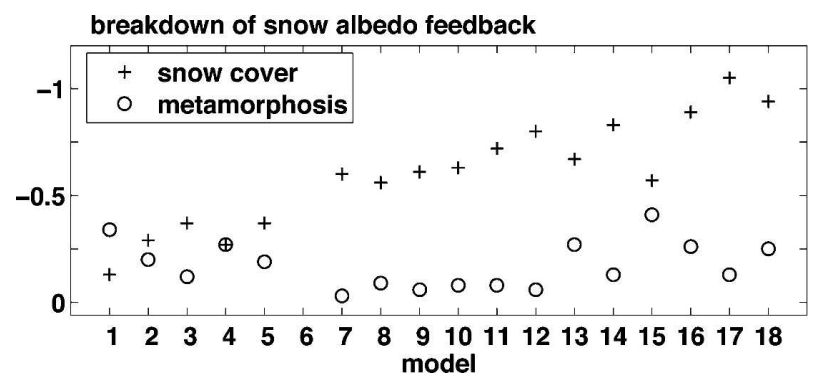

Fig. 4. Breakdown of $\Delta \alpha_{s} / \Delta T_{s}$ into snow cover $\left(\kappa_{2} \Delta S_{c} / \Delta T_{s}\right)$ and snow metamorphosis $\left(\kappa_{3}\right)$ components, represented by "+" and "o" signs, respectively. The PCM values were not shown in this figure because this model did not have either snow cover or snow mass available when our analyses were performed. Units are $\% \mathrm{~K}^{-1}$. 


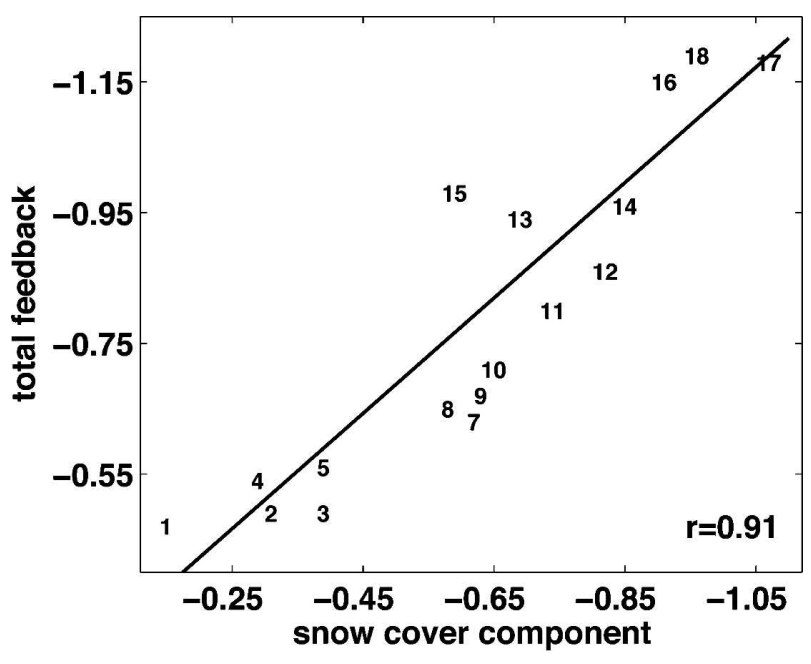

FIG. 5. Scatterplot of $\Delta \alpha_{s} / \Delta T_{s}$ (ordinate) vs $\kappa_{2} \Delta S_{c} / \Delta T_{s}$ (abscissa) in 17 simulations. The numbers of the 17 experiments (Table 1) are used as plotting symbols. A least squares fit regression line is also shown. Units are $\% \mathrm{~K}^{-1}$.

compared to the snow cover component, but it barely contributes to the spread of $\Delta \alpha_{s} / \Delta T_{s}$ seen in Fig. 2. As shown in Fig. 5, the intermodel variations of $\Delta \alpha_{s} / \Delta T_{s}$ are highly correlated with the intermodel variations of the snow cover component. There are a few cases where the snow cover component does not dominate. The metamorphosis component in the Meteorological Research Institute Coupled General Circulation Model version 2.3.2a (MRI CGCM2.3.2; model 1) is twice as large as the snow cover component, while the two components are comparable in the Centre National de Recherches Météorologiques Coupled Global Climate Model version 3 (CNRM-CM3; model 2) and Goddard Institute for Space Studies Model E-R (GISS-ER; model 4).

\section{Controls on the snow cover component}

Because of its dominance of SAF in most models as well as the intermodel spread of the feedback, we wish to decompose the snow cover component further and determine what controls it. According to Eq. (14), two factors govern its magnitude, one being the mean contrast between effective snow albedo and snow-free land albedo $\left(\kappa_{2}\right)$ and the other being the sensitivity of snow cover to $T_{s}\left(\Delta S_{s} / \Delta T_{s}\right)$. The second factor is straightforward to calculate from model output and is shown in Fig. 6a as an average over NH landmasses for each model. To quantify $\kappa_{2}$ we simply divide the snow cover component (Fig. 4) by the $\Delta S_{c} / \Delta T_{s}$ values of Fig. 6a. The $\kappa_{2}$ values are shown in Fig. 6b. Though $\kappa_{2}$ and $\Delta S_{c} / \Delta T_{s}$ both exhibit variability from model to model,
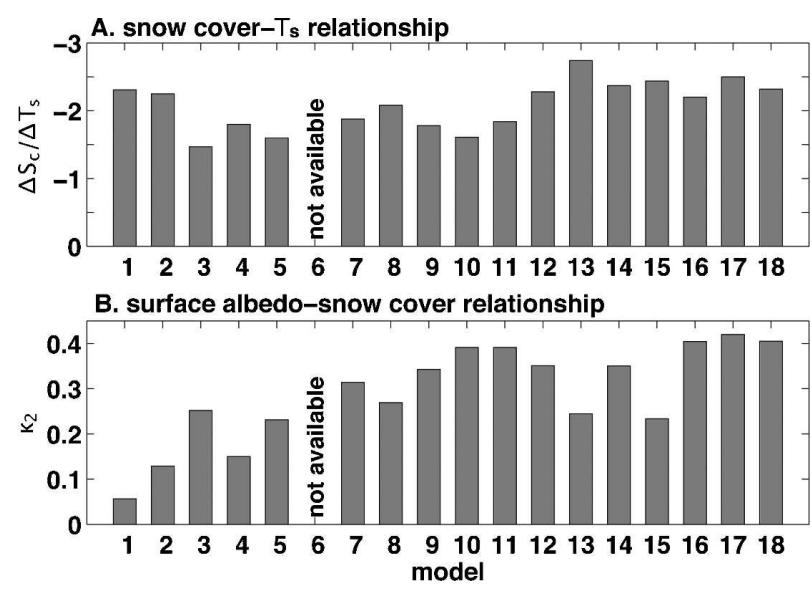

FIG. 6. Values of (a) $\Delta S_{c} / \Delta T_{s}\left(\% \mathrm{~K}^{-1}\right)$ and (b) $\kappa_{2}$ (dimensionless) in 17 simulations.

$\kappa_{2}$ exhibits significantly more intermodel variation. Moreover, the variations of $\kappa_{2}$ are more consistent with the intermodel variations of the total snow cover component than those of $\Delta S_{c} / \Delta T_{s}$. This is confirmed in the scatterplot of $\kappa_{2} \Delta S_{c} / \Delta T_{s}$ vs $\kappa_{2}$ (Fig. 7a). The square of the correlation between $\kappa_{2} \Delta S_{c} / \Delta T_{s}$ and $\kappa_{2}$ is 0.83 . This implies that the contrast between effective snow albedo and snow-free land albedo accounts for about $80 \%$ of the intermodel variance in the snow cover component of SAF, with the relationship between snow cover and $T_{s}$ accounting for the remaining $20 \%$.

To determine what factors are responsible for the intermodel variation in the contrast between effective snow albedo and snow-free land albedo, we average effective snow albedo for the current climate $\left(\alpha_{\text {snow }}^{p}\right)$ and snow-free land albedo $\left(\alpha_{\text {land }}\right)$ over the entire snowcovered region of each model. It turns out that the intermodel variability in $\alpha_{\text {snow }}^{p}$ is much larger than the intermodel variability in $\alpha_{\text {land }}$, so that the intermodel variability in the mean contrast between these two quantities is overwhelmingly dominated by the $\alpha_{\text {snow }}^{p}$ contribution. This is confirmed by a scatterplot of $\kappa_{2}$ against $\alpha_{\text {snow }}^{p}$ (Fig. 7b). The correlation coefficient implies that more than $80 \%$ of the intermodel variance in $\kappa_{2}$ can be accounted for by the variation in the mean albedo of $100 \%$ snow-covered surfaces.

The successive links between the magnitudes of the total SAF $\left(\Delta \alpha_{s} / \Delta T_{s}\right)$, the snow cover component of the feedback $\left(\kappa_{2} \Delta S_{c} / \Delta T_{s}\right)$, the contrast between effective snow albedo and snow-free land albedo $\left(\kappa_{2}\right)$, and finally the mean effective snow albedo itself $\left(\alpha_{\text {snow }}^{p}\right)$ are each strong enough that the mean effective snow albedo is a reasonably good predictor of the total SAF strength. This is clear in the scatterplot of $\Delta \alpha_{s} / \Delta T_{s}$ against $\alpha_{\text {snow }}^{p}$ (Fig. 8). The 2 variables are clearly related, and the 

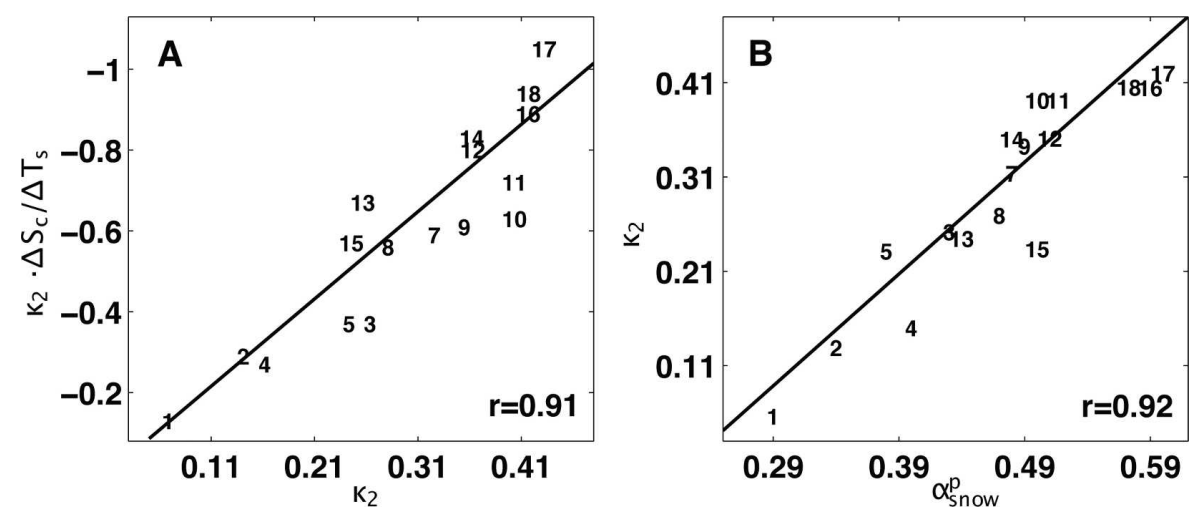

FIG. 7. Scatterplot of (a) $\kappa_{2} \Delta S_{c} / \Delta T_{s}$ (ordinate; units are \% K ${ }^{-1}$ ) vs $\kappa_{2}$ (abscissa; dimensionless) and (b) $\kappa_{2}$ (ordinate; dimensionless) vs mean effective snow albedo for the current climate (abscissa; expressed as a dimensionless fraction) in 17 simulations. The numbers of the $17 \mathrm{ex}-$ periments (Table 1) are used as plotting symbols. Least squares fit regression lines are also shown in (a), (b).

correlation coefficient implies that the intermodel variation in mean effective snow albedo accounts for more than $2 / 3$ of the intermodel variance in SAF strength.

There is a further relationship between mean effective snow albedo and SAF strength, and surface-albedo parameterization, as revealed by the color coding of the model numbers in Fig. 8. Models with the weakest SAF (models $1-5$ ) all use either type 1 or 2 parameteriza-

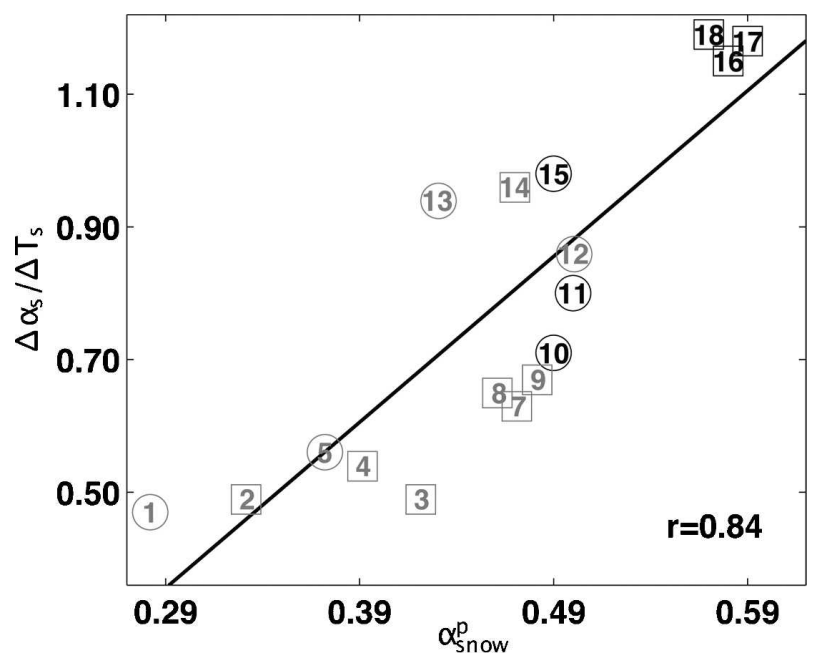

FIG. 8. Scatterplot of $\Delta \alpha_{s} / \Delta T_{s}$ (ordinate; units are $\% \mathrm{~K}^{-1}$ ) vs mean effective snow albedo for the current climate (abscissa; expressed as a dimensionless fraction) in 17 simulations. The numbers of the 17 experiments (Table 1) are used as plotting symbols. A least squares fit regression line is also shown. The numbers are color coded by the way surface albedo is parameterized in snowcovered areas: gray circle $=$ type 1 , gray square $=$ type 2 , black circle $=$ type 3 , and black square $=$ type 4 . See section 2 for an explanation of the four parameterization types. tions (where ground albedo is given as a weighted mean of snow albedo and soil albedo, with weights determined by snow cover, and then overall surface albedo is a weighted mean of canopy albedo and ground albedo with weights given by the fraction of vegetation coverage), and they all have very low mean effective snow albedos. Models with very strong SAF (models 16-18) all use the type 4 parameterization (where overall surface albedo is a weighted mean of snow-free land albedos and snow albedo, with weights determined by snow cover), and they have the highest mean effective snow albedos. The models with type 3 parameterization (models 10,11, and 15), as well as a few models with type 1 and 2 parameterizations (models 7-9 and 12-14), have intermediate mean effective snow albedos, and thus have intermediate SAF.

It might be assumed that the more complex models with explicit treatment of the vegetation canopy have more realistic SAF. However this may not be the case. In a previous study (Hall and Qu 2006), we showed that the simulated strength of SAF in the seasonal cycle context is an excellent predictor of SAF strength in the context of transient climate change. Thus models with the weakest SAF in the climate change context (models 1-5) all have unrealistically weak SAF in the seasonal cycle context by approximately a factor of 2 . These models also all employ type 1 or 2 land surface parameterizations and have low albedos for snow-covered surfaces. We speculate that in these models, either 1) prescribed albedos of snow-covered ground or canopy when snow is present are too low or 2) that vegetation coverage determining the weighting of canopy albedo and ground albedo is too large, placing unrealistic emphasis on the vegetation canopy in the determination of 
overall effective surface albedo when the ground is at least partly snow-covered. At the same time, the models with the strongest SAF in the climate change context (models 16-18) all have unrealistically strong SAF in the seasonal cycle context by about $10 \%-20 \%$, employ type 4 land surface parameterizations, and have very high albedos for snow-covered surfaces. We speculate that in these models snow albedos are assigned based on surface-albedo measurements of snowcovered surfaces with little protruding vegetation, so that completely snow-covered surfaces where vegetation does protrude in the real world are assigned unrealistically large albedos. This interpretation is supported by the fact that type 3 models, distinguished from type 4 models by the fact that snow albedos are prescribed according to vegetation type, have a somewhat lower mean effective snow albedo and a consistently weaker SAF.

\section{Summary and implications}

The strength of SAF in the current generation of transient climate change simulations is determined primarily by the surface-albedo decrease associated with loss of snow cover rather than the reduction in snow albedo due to snow metamorphosis in a warming climate. The large intermodel spread in SAF strength is likewise attributable mostly to the snow cover component. The spread in the strength of this component is in turn mostly attributable to a correspondingly large spread in mean effective snow albedo. Models with large effective snow albedos have a large surfacealbedo contrast between snow-covered and snow-free regions and exhibit a correspondingly large surfacealbedo decrease when snow cover decreases.

Effective snow albedo corresponds roughly with the type of surface-albedo parameterization used. The models without explicit treatment of the vegetation canopy typically have high effective snow albedos and generally have strong SAF. In models with explicit canopy treatment, surfaces that are completely snowcovered typically have lower albedos, and hence the simulations generally have weaker SAF. However, the greater complexity of the latter models does not guarantee greater realism. It is true that the models with the simplest surface-albedo parameterizations have unrealistically strong SAF in the seasonal cycle context, likely attributable to prescribed snow albedos that are too high. However, SAF in models with the most complex parameterizations is significantly weaker than observed. We speculate that in these models either snow albedos or canopy albedos when snow is present are too low, or perhaps more likely, vegetation shields snow- covered surfaces excessively. In any event, detailed, high quality observations of surface albedo in a representative sampling of snow-covered surfaces would be extremely useful in constraining these parameterizations and reducing SAF spread in the next generation of models. This need is particularly emphasized in the recent work of Zhang et al. (2006), who demonstrated the presence of significant discrepancies among the current available surface-albedo datasets in snow-covered areas.

Finally, we note that the relationship between snow cover and $T_{s}$ makes a secondary though nonnegligible contribution to the spread in SAF, accounting for approximately $20 \%$ of the intermodel variability in SAF strength. In this study, $\Delta S_{c} / \Delta T_{s}$ is the $\mathrm{NH}$ average change in snow cover divided by the average change in $T_{s}$ over the same region. The size of the snow cover anomaly associated with a $1^{\circ} \mathrm{C} T_{s}$ anomaly in this case may be somewhat sensitive to the geographical structure of the $\Delta T_{s}$ anomaly, which may in turn be affected by other feedbacks such as cloud- and sea ice-albedo feedback.

Acknowledgments. This research was supported by NSF Grant ATM-0135136. Opinions, findings, conclusions, or recommendations expressed here are those of the authors and do not necessarily reflect NSF views. We thank the international modeling groups for providing data, PCMDI for collecting and archiving this data, JSC/CLIVAR WGCM and their CMIP and Climate Simulation Panel for organizing the model analysis, and the IPCC WG1 TSU for technical support. The IPCC data archive at the Lawrence Livermore National Laboratory is supported by the U.S. Department of Energy. We also thank Alan Robock and one anonymous reviewer for their constructive criticism of this manuscript.

\section{APPENDIX}

\section{Converting Snow Mass to Snow Cover}

Though snow mass per unit area is included in the output of nearly all 18 simulations listed in Table $1, S_{c}$ is given in only 5 of them. Here we use the following technique to convert snow mass to $S_{c}$. At locations with snow mass greater than $60 \mathrm{~kg} \mathrm{~m}^{-2}$, we assume $S_{c}$ to be 1. At locations with snow mass smaller than this threshold, we assume $S_{c}$ to be proportional to snow mass. The coefficient of proportionality is chosen to be the inverse of the threshold so that $S_{c}$ is 1 at locations with snow mass equal to the threshold. This technique is similar to that used by Frei et al. (2003).

We can validate $S_{c}$ obtained from snow mass by this technique in the five simulations providing both $S_{c}$ and 

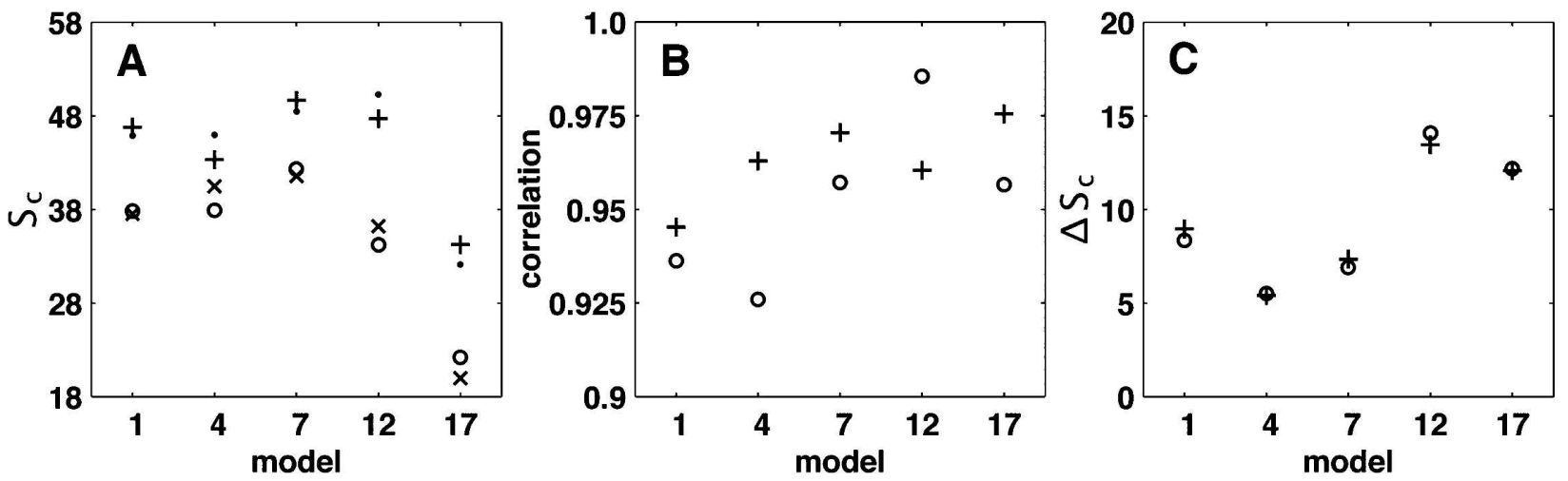

FIG. A1. (a) The climatological springtime mean snow cover (\%) seen in five simulations in the present-day (plus) and future (circle) climate, and that calculated from snow mass in the present-day (point) and future (cross) climate. (b) The correlation between springtime mean snow cover seen in five simulations and that calculated from snow mass (see text) in the present-day (plus) and future (circle) climate. (c) The difference in the climatological springtime mean snow cover (\%) between the present-day and future climate seen in five simulations (plus) and that calculated based on snow mass (circle).

snow mass. First, the climatological springtime mean values of predicted and outputted $S_{c}$ values agree with one another to within $1 \%-2 \%$ in both current and future climates in all 5 simulations (Fig. A1a). Second, there is nearly a perfect correlation between predicted and outputted time series of springtime mean $S_{c}$ values in both the current and future climate (Fig. A1b). And finally, the difference in $S_{c}$ between the two climates calculated from snow mass is almost exactly the same as the $\Delta S_{c}$ values calculated directly from model output (Fig. A1c). The nearly perfect agreement in predicted and outputted climatological mean $S_{c}$, interannual variations in $S_{c}$, and anthropogenic change in $S_{c}$ in all five simulations gives a high degree of confidence in the technique we use to convert snow mass to $S_{c}$ in the other simulations.

\section{REFERENCES}

Alekseev, V. A., E. M. Volodin, V. Y. Galin, V. P. Dymnikov, and V. N. Lykossov, 1998: Modelling of the present-day climate by the atmospheric model of INM RAS "DNM GCM." INM Tech. Rep. N2086-B98, Institute of Numerical Mathematics, Russian Academy of Sciences, 208 pp.

Budyko, M. I., 1969: The effect of solar radiation variations on the climate of the earth. Tellus, 21, 611-619.

Cess, R. D., and G. L. Potter, 1988: A methodology for understanding and intercomparing atmospheric climate feedback processes in general circulation models. J. Geophys. Res., 93 (D7), 8305-8314.

, and Coauthors, 1991: Interpretation of snow-climate feedback as produced by 17 general circulation models. Science, 253, 888-892.

Chalita, S., and H. Le Treut, 1994: The albedo of temperate and boreal forest and the Northern Hemisphere climate: A sensitivity experiment using the LMD GCM. Climate Dyn., 10, 231-240.

Cox, P. M., R. A. Betts, C. B. Bunton, R. L. H. Essery, P. R.
Rowntree, and J. Smith, 1999: The impact of new land surface physics on the GCM simulation of climate and climate sensitivity. Climate Dyn., 15, 183-203.

Douville, H., J.-F. Roye, and J.-F. Mahfouf, 1995: A new snow parameterization for the Météo-France climate model. Climate Dyn., 12, 21-35.

Essery, R., M. Best, and P. Cox, 2001: MOSES 2.2 technical documentation. Hadley Centre Tech. Note 30, 31 pp.

Frei, A., J. A. Miller, and D. A. Robinson, 2003: Improved simulations of snow extent in the second phase of the Atmospheric Model Intercomparison Project (AMIP-2). J. Geophys. Res., 108, 4369, doi:10.1029/2002JD003030.

Gordon, H. B., and Coauthors, 2002: The CSIRO Mk3 Climate System Model. CSIRO Atmospheric Research Tech. Paper 60, $134 \mathrm{pp}$

Hall, A., 2004: The role of surface albedo feedback in climate. $J$. Climate, 17, 1550-1568.

— strain snow albedo feedback in future climate change. Geophys. Res. Lett., 33, L03502, doi:10.1029/2005GL025127.

Hansen, J., G. Russell, D. Rind, P. Stone, A. Lacis, S. Lebedeef, R. Ruedy, and L. Travis, 1983: Efficient three-dimensional global models for climate studies: Models I and II. Mon. Wea. Rev., 111, 609-662.

Milly, P. C. D., and A. B. Shmakin, 2002: Global modeling of land water and energy balances. Part I: The Land Dynamics (LaD) model. J. Hydrometeor., 3, 283-299.

Oleson, K. W., and Coauthors, 2004: Techical description of the Community Land Model (CLM). NCAR Tech. Note NCAR/ TN-464+STR, $186 \mathrm{pp}$.

Qu, X., and A. Hall, 2006: Assessing snow albedo feedback in simulated climate change. J. Climate, 19, 2617-2630.

Randall, D. A., and Coauthors, 1994: Analysis of snow feedbacks in 14 general circulation models. J. Geophys. Res., 99 (D10), $20757-20772$.

Robock, A., 1980: The seasonal cycle of snow cover, sea ice and surface albedo. Mon. Wea. Rev., 108, 267-285.

_ 1983: Ice and snow feedbacks and the latitudinal and seasonal distribution of climate sensitivity. J. Atmos. Sci., 40, 986-997. 
— 1985: An updated climate feedback diagram. Bull. Amer. Meteor. Soc., 66, 786-787.

Roeckner, E., and Coauthors, 1996: The atmospheric general circulation model ECHAM4: Model description and simulation of present-day climate. Max Planck Institute for Meteorology Rep. 218, 90 pp.

, and Coauthors, 2003: The atmospheric general circulation model ECHAM5: Part I: Model description. Max Planck Institute for Meteorology Rep. 349, 140 pp.

Schneider, S. H., and R. E. Dickinson, 1974: Climate modeling. Rev. Geophys. Space Phys., 12, 447-493.

Sellers, W. D., 1969: A global climatic model based on the energy balance of the earth-atmosphere system. J. Appl. Meteor., 8, $392-400$.

Takata, K., S. Emoria, and T. Watanabe, 2003: Development of the minimal advanced treatments of surface interaction and runoff. Global Planet. Change, 38, 209-222.

Verseghy, D. L., N. A. McFarlane, and M. Lazare, 1993: CLASS-A
Canadian land surface scheme for GCMs, II. Vegetation model and coupled runs. Int. J. Climatol., 13, 347-370.

Wiscombe, W. J., and S. G. Warren, 1980: A model for the spectral albedo of snow. I: Pure snow. J. Atmos. Sci., 37, 27122733.

Yu, Y., X. Zhang, and Y. Guo, 2004: Global coupled oceanatmosphere general circulation models in LASG/IAR. $A d v$. Atmos. Sci., 21, 444-455.

Yukimoto, S., and Coauthors, 2006: Present-day climate and climate sensitivity in the Meteorological Research Institute Coupled GCM version 2.3 (MRI-CGCM2.3). J. Meteor. Soc. Japan, 84, 333-363.

Zhang, Y.-C., W. B. Rossow, and P. W. Stackhouse Jr., 2006: Comparison of different global information sources used in surface radiative flux calculation: Radiative properties of the near-surface atmosphere. J. Geophys. Res., 111, D13106, doi:10.1029/2005JD006873. 